\title{
A network-based biomarker approach for molecular investigation and diagnosis of lung cancer
}

\author{
Yu-Chao Wang, Bor-Sen Chen*
}

\begin{abstract}
Background: Lung cancer is the leading cause of cancer deaths worldwide. Many studies have investigated the carcinogenic process and identified the biomarkers for signature classification. However, based on the research dedicated to this field, there is no highly sensitive network-based method for carcinogenesis characterization and diagnosis from the systems perspective.

Methods: In this study, a systems biology approach integrating microarray gene expression profiles and proteinprotein interaction information was proposed to develop a network-based biomarker for molecular investigation into the network mechanism of lung carcinogenesis and diagnosis of lung cancer. The network-based biomarker consists of two protein association networks constructed for cancer samples and non-cancer samples.

Results: Based on the network-based biomarker, a total of 40 significant proteins in lung carcinogenesis were identified with carcinogenesis relevance values (CRVs). In addition, the network-based biomarker, acting as the screening test, proved to be effective in diagnosing smokers with signs of lung cancer.

Conclusions: A network-based biomarker using constructed protein association networks is a useful tool to highlight the pathways and mechanisms of the lung carcinogenic process and, more importantly, provides potential therapeutic targets to combat cancer.
\end{abstract}

\section{Background}

Cancer, the complex disease of uncontrolled cell growth, is one of the leading causes of human death worldwide and the deaths from cancer are projected to continue rising $[1,2]$. Among all types of cancer, the most commonly diagnosed, as well as the most common cause of cancer deaths, is lung cancer, with a mortality rate as high as $80-85 \%$ within 5 years $[1,3]$. Lung cancer is categorized into two main types: small cell lung carcinoma (SCLC) and non-small cell lung carcinoma (NSCLC). NSCLCs are subcategorized into three main subtypes: squamous cell carcinoma, adenocarcinoma, and large cell carcinoma [4]. Previous research has shown that these major histological types of lung cancer are associated with cigarette smoking [5]. In light of this, much research has been devoted to investigating the molecular

\footnotetext{
* Correspondence: bschen@ee.nthu.edu.tw

Laboratory of Control and Systems Biology, Department of Electrical Engineering, National Tsing Hua University, Hsinchu 30013, Taiwan
}

alterations which ensued from cigarette smoking and the mechanism that links cigarette smoking to lung cancer. Spira et al. used DNA microarray to compare the gene expressions of large-airway epithelial cells from nonsmokers and smokers, and to determine how cigarette smoking alters the transcriptome [6]. Hecht indicated that many tobacco smoke carcinogens, such as polycyclic aromatic hydrocarbons and nicotine-derived nitrosamine ketone are predominant inducers of lung cancer [7]. Recently, Takahashi et al. showed that induction of IKK $\beta$ - and JNK1-dependent inflammation is likely to be an important contributor to the tumorpromoting activity of tobacco smoke [8].

In addition to the investigation on carcinogenesis, many studies identified cancer biomarkers through analysis of genome-wide expression profiles $[9,10]$. The biomarkers are used either as a diagnostic evaluation to determine the health of a patient with or without the cancer, or as a prognostic indicator to determine the patient's prognosis. Spira et al. used gene expression 
profiles of samples from lung cancer patients to identify an 80-gene biomarker that distinguished apparent differences between smokers with and without lung cancer [3]. Because there is no effective screening tool for diagnosing lung cancer at an early stage, the 80 -gene biomarker could make a beneficial contribution to minimizing high mortality rates by providing a better prognosis $[3,11]$. However, the biomarker identification method, which strictly uses gene expression profiles, cannot show how the different genes within the biomarker gene set are related to each other, i.e., the biomarkers are not identified from the systems perspective. Further, the gene lists obtained for similarly diagnosed patients by different research groups differ widely and share few common genes [12].

Due to these kinds of limitations and the widely accepted opinion that cancer is a disease of pathways $[13,14]$, both protein-protein interaction (PPI) and pathway information are integrated for biomarker identification. Chuang et al. developed a protein-network-based approach that identifies biomarkers not as individual genes but as sub-networks extracted from protein interaction databases. They showed that the sub-network classification is highly accurate in signature discrimination and provide an accurate account of the network structure [15]. Many other network-based approaches for prioritizing disease genes and protein interaction subnetworks that are discriminative of disease signature have been developed [16-19]. The dynamic structure of the human protein interaction network has recently been examined to aid in predicting breast cancer prognosis, suggesting that network modularity might be a defining feature of tumor phenotype [20].

Network analysis has shown that under different cellular states or in response to diverse stimuli, transcription factors alter their interactions to regulate different genes, thereby rewiring the network [21]. The same situation occurs with protein interaction networks $[20,22]$. Motivated by the dynamic structure of the human protein interaction network and the observation that interacting proteins tend to result in similar disease phenotypes when dysregulated [23], we developed a computational framework to construct the networkbased biomarker for molecular investigation and diagnosis of lung cancer. The network-based biomarker consisted of two protein association networks for cancer and non-cancer smokers. Based on the concept of network comparison [24], 40 significant proteins that play potentially important roles in lung carcinogenesis were identified. The network-based biomarker is a useful tool for further distinguishing the presence of cancer in smokers by similarity measurement of molecular patterns. Hopefully, the proposed method can aid in further understanding lung carcinogenesis and providing potential drug targets for humans to combat lung cancer.

\section{Methods \\ Overview of the network-based biomarker approach for lung cancer investigation}

The overall flowchart of the proposed network-based biomarker approach is shown in Figure 1. Our goal is to investigate lung cancer by the construction of networkbased biomarkers composed of protein association networks for smokers with and without cancer. Microarray gene expression profiles of patient samples and proteinprotein interaction information were integrated for protein selection and network construction. Two protein association networks with quantitative protein association abilities for cancer and non-cancer smokers were constructed. By comparing two protein association networks within the network-based biomarker, a carcinogenesis relevance value (CRV) was computed to correlate proteins with the level of lung carcinogenesis. A higher score suggests the particular protein plays a more critical role in lung carcinogenesis. A set of significant proteins was selected based on the CRV for each protein and the statistical assessment. Further, using microarray data for smokers suspected of having cancer, it is possible to compute mapping errors for further diagnostic evaluation of smokers with or without cancer.

\section{Data selection and preprocessing}

In this study, two kinds of data, microarray gene expression profile and protein-protein interaction information, were integrated. The microarray data were downloaded from the GEO database http://www.ncbi.nlm.nih.gov/ geo/ (accession number GSE4115). Spira et al. performed gene expression profiling in histologically normal large-airway epithelial cells obtained by bronchoscopy of current and former smokers. The health of each individual was recorded following the bronchoscopy until a final diagnosis of either presence or absence of lung cancer [3]. Data were collected from a total pool of 187 subjects and divided into primary and prospective data sets: 79 and 73 smokers with and without lung cancer respectively in the primary data set; 18 and 17 smokers with and without lung cancer respectively in the prospective data set. The primary data set was used for network-based biomarker construction and the prospective data set was used for diagnostic evaluation. Protein-protein interaction (PPI) data were extracted from the Biological General Repository for Interaction Datasets (BioGRID) http://thebiogrid.org/ and the Human Protein Reference Database (HPRD) http://www.hprd.org/. The BioGRID database was developed to house and distribute collections of protein and genetic interactions from major model organism species. 


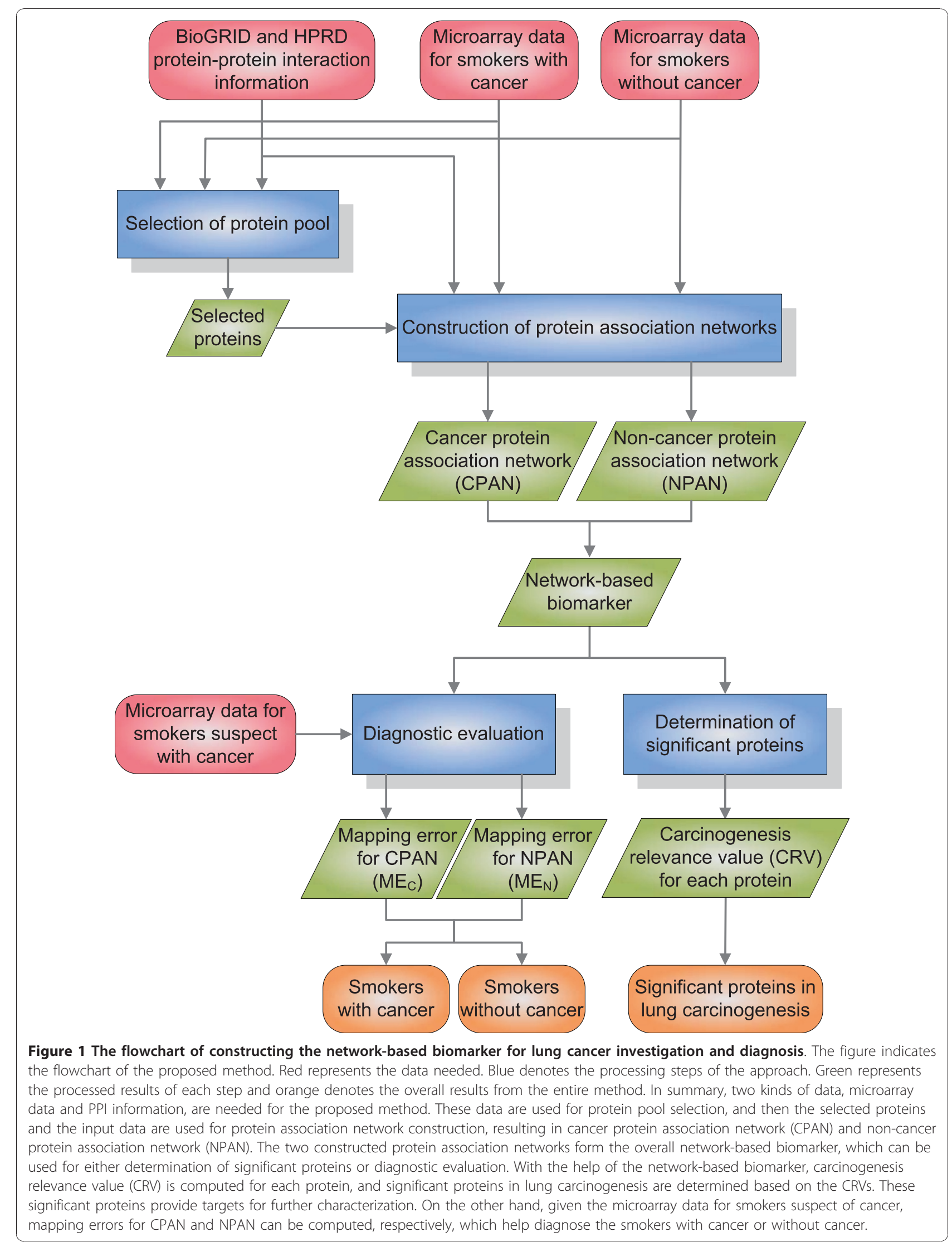


BioGRID currently contains over 340,000 interactions derived from both high-throughput studies and conventional focused studies [25]. The HPRD is a database that integrates a wealth of information relevant to the human proteome, including protein-protein interactions, post-translational modifications, disease associations, and tissue expression [26]. Prior to further processing, the gene expression value $g_{i j}$ is normalized to z-transformed scores $z_{i j}$ so that for each gene $i$ the normalized expression value has mean $\mu_{i}=0$ and standard deviation $\sigma_{i}=1$ over sample $j$.

\section{Selection of protein pool and construction of network- based biomarker}

To integrate the gene expression and PPI information data and construct the network-based biomarker consisting of protein association networks, the expression value of each gene was first overlaid on its corresponding protein. The gene expression for each protein was then used to select differentially expressed proteins using one-way analysis of variance (ANOVA), where the null hypothesis is that the average expression levels for the protein are the same for smokers with and without cancer. The proteins with Bonferroni adjusted $p$-values less than 0.05 were selected for the protein pool. Because we used the network-based biomarker as an investigative tool, the differentially expressed proteins without PPI information were excluded from the protein pool, while proteins based on PPI information that were highly connected with pool proteins were also included. In other words, the protein pool consisted of both differentially expressed proteins and the proteins that are highly connected with them. Based on the protein pool and PPI information, a rough PPI network can be easily constructed by linking proteins that share interactions. It is worth noting that since the data for cancer and non-cancer samples are limited, the number of proteins selected for rough PPI network construction is also restricted. That is, to avoid overfitting in network construction, the maximum degree of the proteins in the rough PPI network should be less than the cancer/noncancer sample number, thereby restricting the size of the rough PPI network.

Using a simple regression model, the rough PPI network was further refined with the microarray data to highlight the independent protein association for samples with and without lung cancer relative to their respective data sets. For a target protein $i$ in the rough PPI network, the protein was described using the following protein association model:

$$
y_{i}[n]=\sum_{k=1}^{N_{i}} \alpha_{i k} y_{i k}[n]+\varepsilon_{i}[n]
$$

where, $y_{i}[n]$ represents the gene expression level of the target protein $i$ for the sample $n ; \alpha_{i k}$ denotes the association ability between the target protein $i$ and its $k^{\text {-th }}$ interactive protein, which quantifies the expression relation between the interactive proteins and can be identified using our data; $y_{i k}[n]$ indicates the gene expression level of the $k^{\text {-th }}$ protein that interacts with the target protein $i$ for sample $n ; N_{i}$ represents the number of proteins interacting with the target protein $i$ and can be obtained from the rough PPI network; and $\varepsilon_{i}[n]$ denotes stochastic noise associated with other factors or model uncertainty. Equation (1) states that, biologically, the expression level of the target protein $i$ is associated with the expression levels of interacting proteins. A protein association model was constructed for each protein in the protein pool.

After the protein association model of the rough PPI network was constructed, the association parameters in equation (1) were identified using maximum likelihood estimation method $[27,28]$ using microarray data (see Additional file 1 for details). Since there are two data sets of microarray data (smokers with and without cancer), the association parameters were identified separately for the cancer data set and non-cancer data set, resulting in $\alpha_{i k, \mathrm{C}}$ and $\alpha_{i k, \mathrm{~N}}$, respectively. In this case, for each protein in each phenotype, i.e., with cancer or without cancer, a mathematical description was constructed to characterize the respective expression association. Once the association parameters for all proteins in the rough PPI network were identified, the significant protein associations were determined based on the estimated association abilities $\left(\alpha_{i k}\right.$ 's). Akaike Information Criterion (AIC) $[27,29]$ and Student's ttest [30] were employed for model order selection and for determining significance of protein associations (see Additional file 1 for details). In doing so, the rough PPI network was refined and the protein association networks for smokers with or without cancer were constructed.

Based on the identified protein association abilities, two matrices were established to represent the cancer protein association network (CPAN) and the non-cancer protein association network (NPAN).

$$
\begin{aligned}
C & =\left[\begin{array}{cccc}
\alpha_{11, \mathrm{C}} & \alpha_{12, \mathrm{C}} & \cdots & \alpha_{1 K, \mathrm{C}} \\
\alpha_{21, \mathrm{C}} & \alpha_{22, \mathrm{C}} & \cdots & \alpha_{2 K, \mathrm{C}} \\
\vdots & \vdots & \ddots & \vdots \\
\alpha_{K 1, \mathrm{C}} & \alpha_{K 2, \mathrm{C}} & \cdots & \alpha_{K K, \mathrm{C}}
\end{array}\right] \\
N & =\left[\begin{array}{cccc}
\alpha_{11, \mathrm{~N}} & \alpha_{12, \mathrm{~N}} & \cdots & \alpha_{1 K, \mathrm{~N}} \\
\alpha_{21, \mathrm{~N}} & \alpha_{22, \mathrm{~N}} & \cdots & \alpha_{2 K, \mathrm{~N}} \\
\vdots & \vdots & \ddots & \vdots \\
\alpha_{K 1, \mathrm{~N}} & \alpha_{K 2, \mathrm{~N}} & \cdots & \alpha_{K K, \mathrm{~N}}
\end{array}\right]
\end{aligned}
$$


where, $\alpha_{i j, \mathrm{C}}$ and $\alpha_{i j, \mathrm{~N}}$ indicate the quantitative protein association ability between protein $i$ and protein $j$ for CPAN and NPAN, respectively; and $K$ is the number of proteins in the protein association network. For a given protein $i$ and protein $j$ in the protein association network, the association ability $\alpha_{i j}$ quantifies the expression relation between the interactive proteins. If $\alpha_{i j}=0$, there is no association between protein $i$ and protein $j$. Further, we said that protein $i$ is associated with protein $j$ means that the expression level changes of protein $i$ account for the expression level changes of protein $j$ and vice versa. As a consequence, when the estimated protein association ability $\alpha_{i j} \neq \alpha_{j i}$, the one which has larger absolute value would be selected as the association ability between protein $i$ and protein $j$, i.e., $\alpha_{i j}=\alpha_{j i}$. The resulting CPAN and NPAN constituted the network-based biomarker used for identifying the significant proteins in lung carcinogenesis and diagnostic evaluation.

\section{Determination of significant proteins in lung carcinogenesis via the network-based biomarker}

According to equations (1) and (2), the protein association models for CPAN and NPAN can be represented as the following equations.

$$
\begin{aligned}
& Y_{\mathrm{C}}=C Y_{\mathrm{C}}+E_{\mathrm{C}} \\
& Y_{\mathrm{N}}=N Y_{\mathrm{N}}+E_{\mathrm{N}}
\end{aligned}
$$

where $\quad Y_{\mathrm{C}}=\left[\begin{array}{llll}y_{1, \mathrm{C}}[n] & y_{2, \mathrm{C}}[n] & \cdots & y_{K, \mathrm{C}}[n]\end{array}\right]^{T}$, $Y_{\mathrm{N}}=\left[\begin{array}{llll}y_{1, \mathrm{~N}}[n] & y_{2, \mathrm{~N}}[n] & \cdots & y_{K, \mathrm{~N}}[n]\end{array}\right]^{T}$ denotes the vectors of expression levels; and $E_{\mathrm{C}}$ and $E_{\mathrm{N}}$ indicate the noise vectors in cancer and non-cancer cases, respectively. A matrix indicating the difference between two protein association networks is defined as $C-N$.

$$
\begin{aligned}
D & =\left[\begin{array}{cccc}
d_{11} & d_{12} & \cdots & d_{1 K} \\
d_{21} & d_{22} & \cdots & d_{2 K} \\
\vdots & \vdots & \ddots & \vdots \\
d_{K 1} & d_{K 2} & \cdots & d_{K K}
\end{array}\right] \\
& =\left[\begin{array}{cccc}
\alpha_{11, \mathrm{C}}-\alpha_{11, \mathrm{~N}} & \alpha_{12, \mathrm{C}}-\alpha_{12, \mathrm{~N}} & \cdots & \alpha_{1 K, \mathrm{C}}-\alpha_{1 K, \mathrm{~N}} \\
\alpha_{21, \mathrm{C}}-\alpha_{21, \mathrm{~N}} & \alpha_{22, \mathrm{C}}-\alpha_{22, \mathrm{~N}} & \cdots & \alpha_{2 K, \mathrm{C}}-\alpha_{2 K, \mathrm{~N}} \\
\vdots & \vdots & \ddots & \vdots \\
\alpha_{K 1, \mathrm{C}}-\alpha_{K 1, \mathrm{~N}} & \alpha_{K 2, \mathrm{C}}-\alpha_{K 2, \mathrm{~N}} & \cdots & \alpha_{K K, \mathrm{C}}-\alpha_{K K, \mathrm{~N}}
\end{array}\right]
\end{aligned}
$$

where $d_{i j}$ denotes the difference in protein association ability between CPAN and NPAN among protein $i$ and protein $j$. Using the matrix $D$ to show the difference in network structure between CPAN and NPAN, a carcinogenesis relevance value (CRV) was presented to quantify the correlation of each protein with significance of lung carcinogenesis. To identify the significant proteins in lung carcinogenesis, two important issues were taken into consideration. First, the magnitude of the association abilities $\alpha_{i j}$ 's denotes the significance of one protein to the other one. A higher absolute value of $\alpha_{i j}$ implies that the two proteins are more tightly associated. Second, if a protein plays a more crucial role in lung carcinogenesis, the difference in association numbers linked to the protein for CPAN and NPAN would be larger. For example, if one protein shares a strong association with many proteins in CPAN, but a weaker association (no protein) in NPAN, the protein in question is more likely involved in lung carcinogenesis. As a result, the CRV was determined based on the difference in protein association abilities via the following equation.

$$
\mathrm{CRV}_{i}=\sum_{j=1}^{K}\left|d_{i j}\right|
$$

For the $i^{\text {th }}$ protein in the network-based biomarker, the implication of equation (5) is that the CRV quantifies the extent of protein associations that differentiate CPAN from NPAN.

In addition to the CRV assigned, an empirical $p$-value was also obtained for each protein to determine the statistical significance of the CRV. To determine the $p$ value for an observed CRV, a null distribution of CRVs (Figure 2) was generated by repeatedly permuting the network structure of the rough PPI network and computing the CRV for each random network structure. The permutation of the network structure was performed by maintaining network size, i.e., interacted proteins were permuted without altering the total number of protein interactions. The process was repeated 100,000 times and the $p$-value of the corresponding CRV was estimated as the fraction of random network structures whose CRV is at least as large as the CRV of the real network structure. The CRVs with $p$-value $\leq$ 0.05 were determined as significant CRVs and the corresponding proteins were identified as significant proteins in lung carcinogenesis.

\section{Diagnostic evaluation by the network-based biomarker}

An important feature of the proposed network-based biomarker approach is its ability to identify significant proteins in lung cancer, and perform accurate diagnosis of smokers suspect of lung cancer. Using the new microarray expression data for smokers, we can classify a sample into smoker with cancer or smoker without cancer based on CPAN and NPAN within the networkbased biomarker. The idea comes from the similarity comparison of new sample data between CPAN and NPAN. Specifically, sample data that are more similar to the network structure of CPAN than to NPAN signifies 


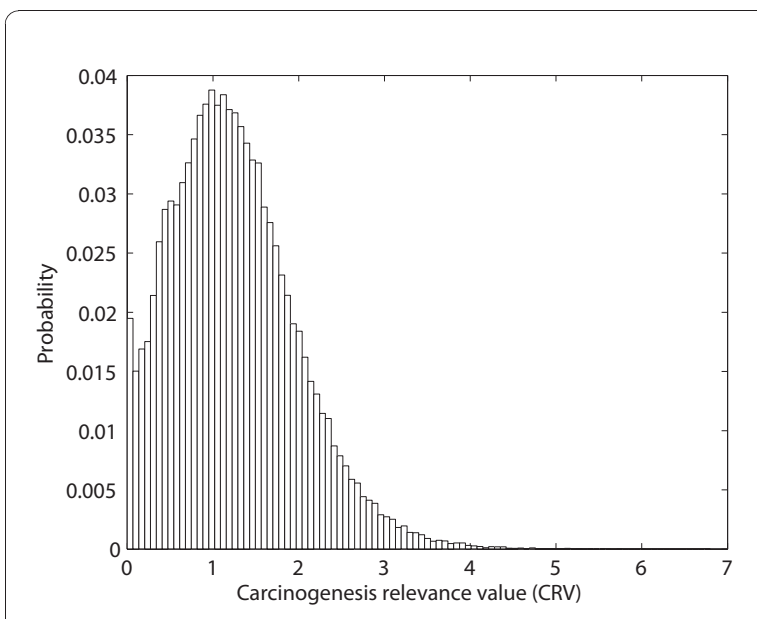

Figure 2 Distribution of carcinogenesis relevance values (CRVs) of random networks. The null distribution of CRVs is generated by 100,000 randomly permuted network structures.

the presence of lung cancer in the smoker, and vice versa. Because the network construction uses more than one sample, the new sample data were mapped to the CPAN and NPAN identified above, with the mapping error employed as the criteria for classification. Assuming we had new sample data $Z=\left[\begin{array}{llll}z_{1} & z_{2} & \cdots & z_{K}\end{array}\right]^{T}$ from a smoker, based on equations (1) and (3), the mapping errors for CPAN and NPAN, respectively, are defined as

$$
\begin{aligned}
& \mathrm{ME}_{\mathrm{C}}=\|Z-C \cdot Z\|_{2} \\
& \mathrm{ME}_{\mathrm{N}}=\|Z-N \cdot Z\|_{2}
\end{aligned}
$$

$$
\text { where } \quad\|P\|_{2}=\left(\sum_{i=1}^{K} p_{i}^{2}\right)^{1 / 2}
$$

when

$P=\left[\begin{array}{llll}p_{1} & p_{2} & \cdots & p_{K}\end{array}\right]^{T}$. The mapping errors can be considered as the similarity measurement of the new sample $Z$ to the CPAN and NPAN systems. The smaller the mapping error, the stronger the correlation between the sample data and the protein association network. Consequently, if $\mathrm{ME}_{\mathrm{C}}<\mathrm{ME}_{\mathrm{N}}$, the new sample $Z$ is more similar to the cancer system and is categorized into smokers with cancer, and vice versa. The criteria of mapping errors have simultaneously taken into account the protein association network structures with quantitative association abilities and the expression levels of the proteins. Further, since the modeling error operates as the criterion of classification, the classification is more dependent on network structure than data alone and thus could also be suitable for classification using independent data. We believe that this kind of classification approach can provide new perspective for diagnostic evaluation.

\section{Results}

Construction of network-based biomarker and identification of significant proteins in lung carcinogenesis

We applied the proposed network-based biomarker approach for molecular investigation and diagnosis of lung cancer. The primary data set $(79$ smokers with lung cancer and 73 smokers without lung cancer) of GSE4115 downloaded from the GEO database http:// www.ncbi.nlm.nih.gov/geo/ was used for construction of the network-based biomarker. Based on ANOVA, 199 proteins with PPI information were identified as the differentially expressed proteins and were selected in the protein pool. In addition, the proteins that linked to three differentially expressed proteins in the protein pool according to PPI information were also included in the pool. As a result, the protein pool consisted of 339 proteins. Following this, proteins with PPI information among them were linked together, resulting in the rough PPI network. The expression profiles for smokers with or without cancer and the protein association model (1) were further employed to refine the rough PPI network. The CPAN and NPAN, which consisted of 399 and 393 protein associations respectively, would constitute the network-based biomarker of lung cancer (Figure 3). The difference between CPAN and NPAN is expressed further in Figure 4. According to the CPAN and NPAN with quantitative association abilities, the CRVs for each protein were computed and the significance of these CRVs was determined. Consequently, 40 identified proteins played significant roles in lung carcinogenesis (Table 1 and Additional file 2).

\section{Investigation into the significant proteins in lung carcinogenesis}

The 40 identified proteins contributing to lung carcinogenesis can be divided into three categories according to the functional annotations (Table 1); the three functional subnetworks are shown in Figure 5. The mechanisms in carcinogenesis using the significant proteins were further investigated through various cell life cycle stages.

\section{(1) Cell growth}

Cancer is a complex disease of uncontrolled cell growth. Therefore, the proteins responsible for cell growth are likely to play critical roles in lung carcinogenesis. Among the 40 significant proteins identified based on the network-based biomarker, 30 proteins are annotated with cell growth (Table 1). The mitogenactivated protein kinase (MAPK) cascade is a highly conserved module that is identifiable in many cancers. Three MAPK-related proteins annotated with cell growth, MAPK1 (ERK2), MAPK3 (ERK1) and MAPK14 $(\mathrm{p} 38 \alpha)$, were identified as significant proteins in 


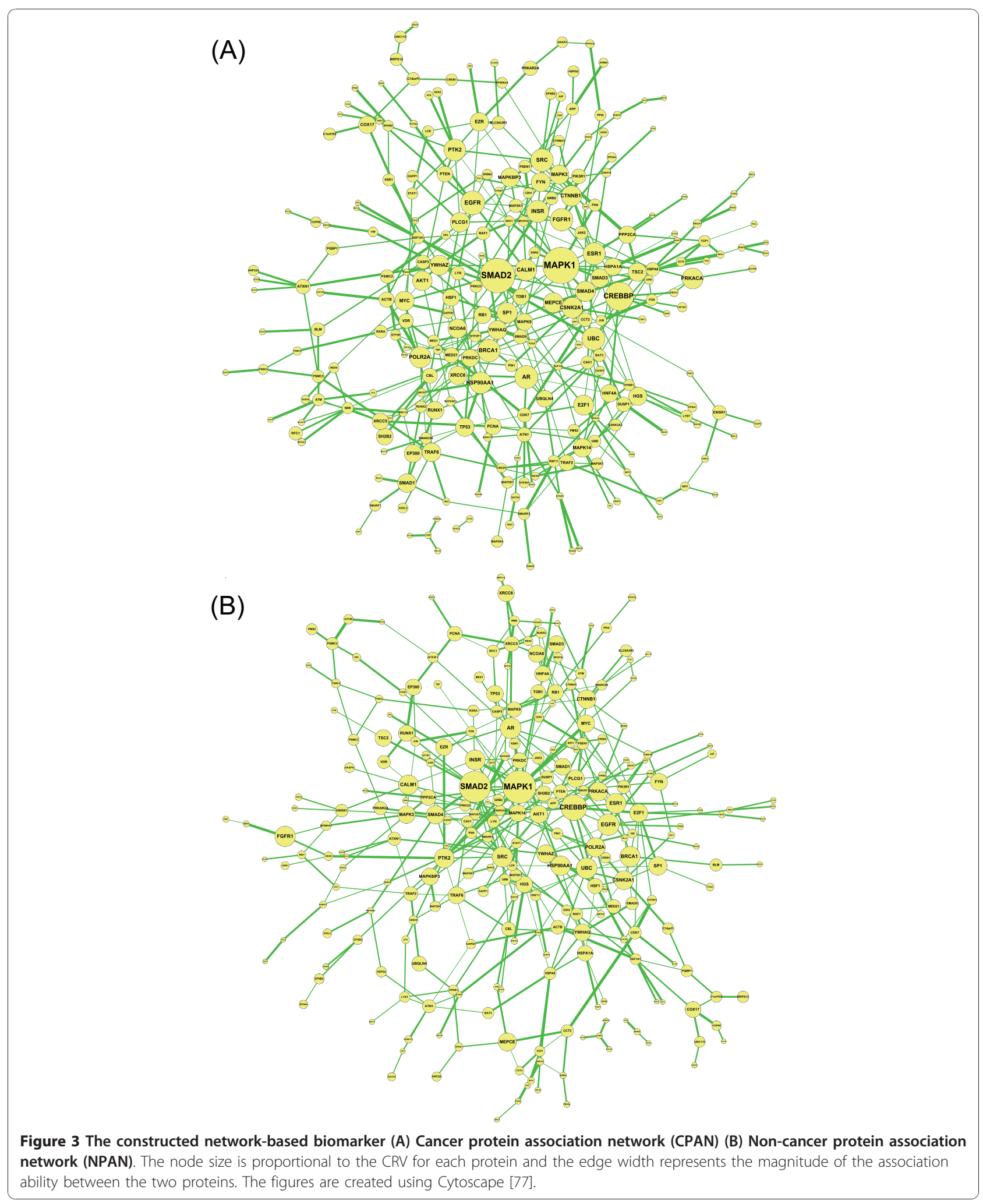




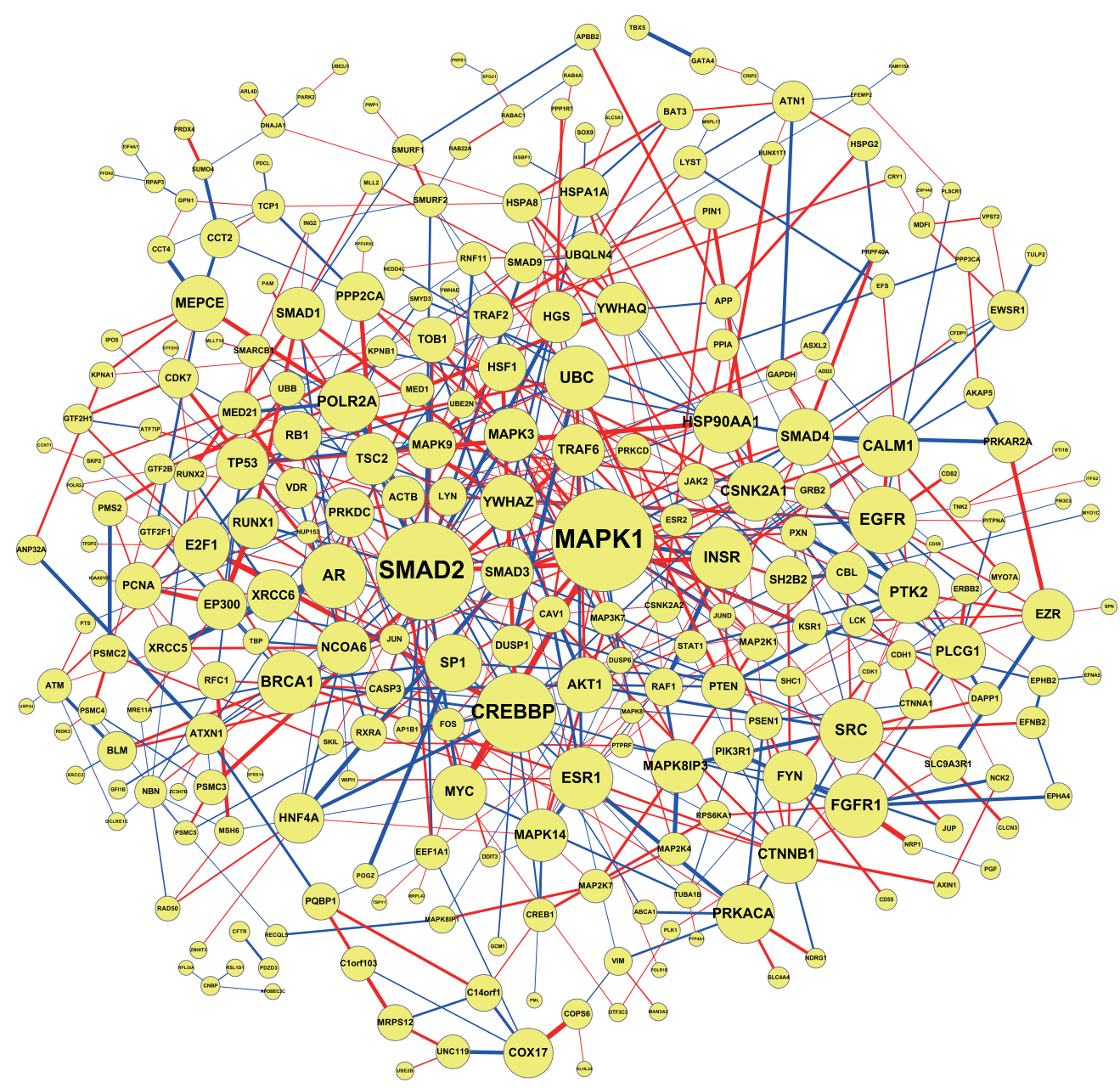

Figure 4 The difference between CPAN and NPAN. The node size is proportional to the CRV for each protein and the edge width represents the magnitude of the association ability between the two proteins. Red and blue edges indicate the positive and negative values of $d_{i j}$ 's in equation (4), respectively. The figure is created using Cytoscape [77].

lung carcinogenesis. Elevated expression of activated MAPK1/3 have been observed in NSCLC [31] and may play a role in lung metastasis [32]. MAPK14 (p38 $\alpha)$ is well known for its important role in response to inflammation and environmental stress [33]; its protein expression is more than three times lower in human lung tumors compared with normal human lung tissue, suggesting that MAPK14 may function as a negative regulator of lung carcinogenesis [34]. Four proteins from the SMAD protein family (SMAD1, SMAD2, SMAD3 and SMAD4) were also identified as significant proteins in lung carcinogenesis. The SMAD proteins, which consist of three functional classes, are signal transducers and transcriptional modulators [35]. Mutations and altered expression for these four proteins were observed in human cancer [36,37], highlighting the significant, albeit varied, roles of the SMAD protein family in carcinogenesis.
EGFR, FGFR1, and INSR are receptor tyrosine kinases (RTKs) bound by an epidermal growth factor, a fibroblast growth factor, and insulin, respectively. Receptor tyrosine kinases have been shown to not only perform a key regulatory role in normal cellular processes, but also become critically involved in the development and progression of human cancers [38]. EGFR is one of the most extensively studied proteins in carcinogenesis. It is overexpressed in NSCLC as well as in other common tumors, and its increased expression is associated with aggressive tumor growth and therapy resistance [39]. EGFR has also been found to be significantly mutated in lung adenocarcinoma $[40,41]$. Because of its significance, EGFR becomes a popular therapeutic target for carcinogenesis. Gefitinib (Iressa) and erlotinib (Tarceva) are two targeted therapies that specifically inhibit EGFR tyrosine kinase [39,41]. FGFR1 and INSR are both known to be involved in carcinogenesis and thus become novel, 
Table 1 The identified significant proteins in lung carcinogenesis

\begin{tabular}{|c|c|c|c|c|c|c|}
\hline \multirow[t]{2}{*}{ Protein symbol $^{\dagger}$} & \multirow[t]{2}{*}{ CRV } & \multirow[t]{2}{*}{$p$-value } & \multicolumn{3}{|c|}{ Functional annotation* } & \multirow[t]{2}{*}{ Literature evidence $^{\S}$} \\
\hline & & & Cell growth & Cell survival & Cell migration & \\
\hline MAPK1 & 8.3418 & $<1 \mathrm{e}-5$ & + & + & + & {$[31,32]$} \\
\hline SMAD2 & 7.7901 & $<1 \mathrm{e}-5$ & + & + & + & {$[36]$} \\
\hline CREBBP & 5.7870 & 0.00002 & + & & & [44] \\
\hline EGFR & 4.3635 & 0.00086 & + & + & + & [39-41] \\
\hline$A R$ & 4.0966 & 0.00159 & + & + & + & [49] \\
\hline UBC & 4.0331 & 0.00180 & & & & \\
\hline SRC & 3.9446 & 0.00218 & + & + & + & {$[51,63]$} \\
\hline FGFR1 & 3.9227 & 0.00237 & + & & + & {$[42]$} \\
\hline BRCA1 & 3.9049 & 0.00243 & + & + & & [58] \\
\hline ESR1 & 3.8409 & 0.00295 & + & + & + & {$[50]$} \\
\hline INSR & 3.7946 & 0.00329 & + & & + & [43] \\
\hline PTK2 & 3.6758 & 0.00432 & + & + & + & {$[63,64]$} \\
\hline HSP90AA1 & 3.6732 & 0.00436 & + & + & + & [54] \\
\hline CALM1 & 3.6363 & 0.00482 & & + & & \\
\hline POLR2A & 3.5701 & 0.00547 & & & & \\
\hline CSNK2A1 & 3.4128 & 0.00761 & + & + & & {$[61]$} \\
\hline PRKACA & 3.3688 & 0.00856 & & + & & \\
\hline CTNNB1 & 3.2935 & 0.00994 & + & + & + & {$[45]$} \\
\hline SP1 & 3.2397 & 0.01133 & + & + & & [59] \\
\hline SMAD4 & 3.1947 & 0.01266 & + & + & + & [36] \\
\hline $\mathrm{E} 2 \mathrm{~F} 1$ & 3.1382 & 0.01407 & + & + & & [46] \\
\hline YWHAZ & 3.1212 & 0.01467 & + & & & [52] \\
\hline MEPCE & 3.0968 & 0.01545 & & & & \\
\hline AKT1 & 3.0193 & 0.01857 & + & + & + & [62] \\
\hline PLCG1 & 2.9654 & 0.02069 & & & + & [66] \\
\hline MYC & 2.8987 & 0.02385 & + & + & & {$[47]$} \\
\hline MAPK3 & 2.8545 & 0.02654 & + & + & + & {$[31,32]$} \\
\hline NCOA6 & 2.8132 & 0.02892 & + & + & & \\
\hline FYN & 2.7833 & 0.03089 & + & & + & [48] \\
\hline MAPK8IP3 & 2.7746 & 0.03141 & & & + & \\
\hline YWHAQ & 2.7582 & 0.03242 & + & & & [53] \\
\hline TRAF6 & 2.7150 & 0.03535 & & + & & {$[57]$} \\
\hline SMAD1 & 2.6940 & 0.03697 & + & + & + & [37] \\
\hline SMAD3 & 2.6815 & 0.03815 & + & + & + & [36] \\
\hline MAPK14 & 2.6727 & 0.03894 & + & + & + & [34] \\
\hline TP53 & 2.6522 & 0.04056 & + & + & + & {$[40,41,55]$} \\
\hline XRCC6 & 2.6270 & 0.04263 & & + & & \\
\hline EZR & 2.6213 & 0.04314 & & & + & [67] \\
\hline TSC2 & 2.6116 & 0.04401 & + & + & + & {$[65]$} \\
\hline HGS & 2.5730 & 0.04744 & + & & & \\
\hline
\end{tabular}

${ }^{\dagger}$ The full names of these proteins according to UniProt database http://www.uniprot.org/ are listed in Additional file 2.

* The functional annotations are from the Gene Ontology database http://www.geneontology.org/ and literatures.

$\S$ The literature evidences indicate that overexpression/dysregulation of the specific protein or mutation of the corresponding gene would result in carcinogenesis.

attractive targets for cancer therapeutic strategies $[42,43]$.

Many proteins acting as transcriptional regulators were also identified. CREBBP is a transcriptional co-activator downstream of the TGF $\beta$ pathway and the mutations and deletions of the CREBBP gene are associated with lung cancer [44]. CTNNB1 ( $\beta$-catenin) is one of the core components in the Wnt pathway. Mutation of $\beta$-catenin, which results in aberrant activation of the Wnt pathway, is a frequent cause of human cancer growth [45]. E2F1 is one of the significant proteins involved in the cell cycle. Its overexpression has been demonstrated in both NSCLC 


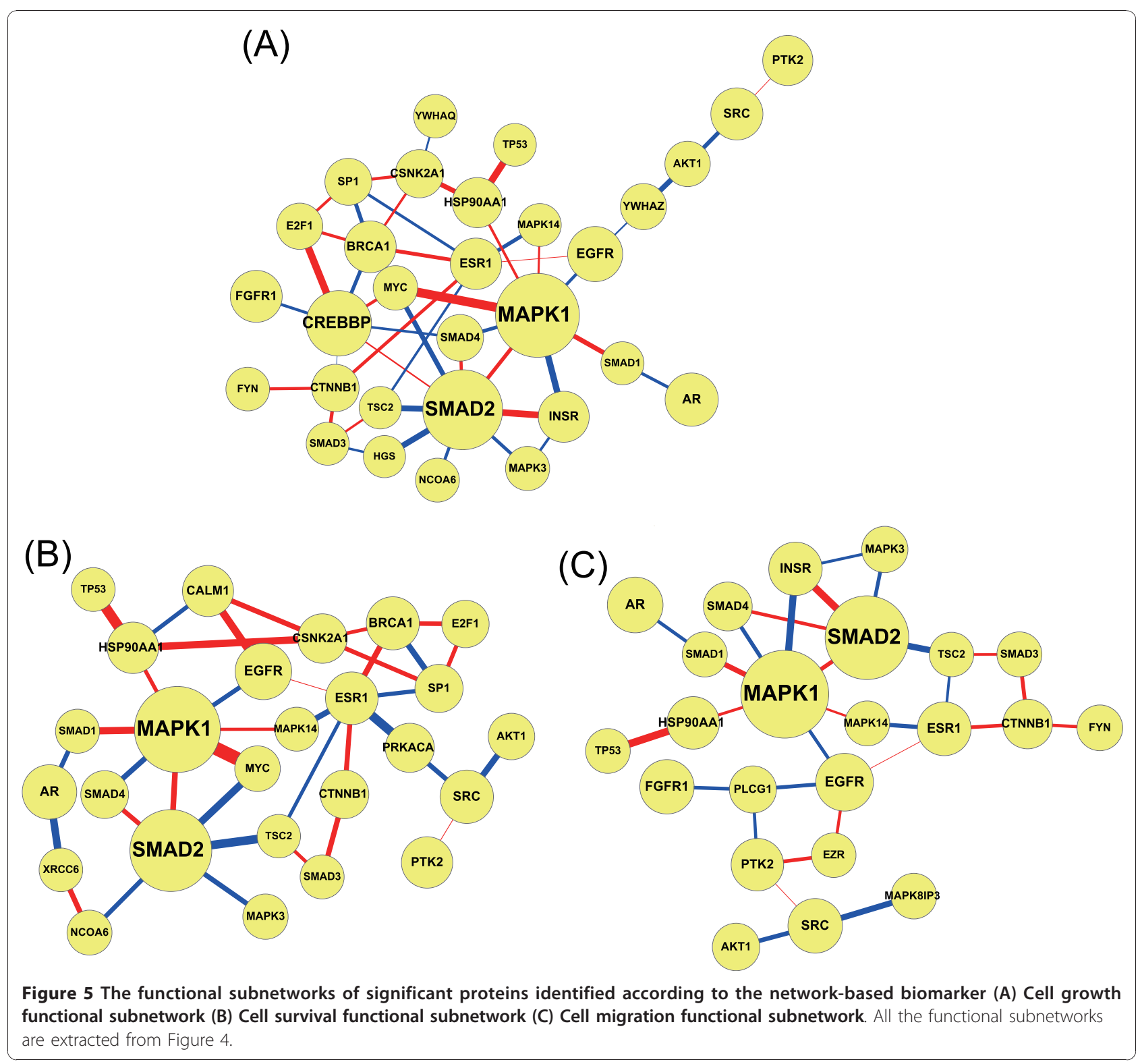

and SCLC and is induced by its upstream RB protein [46]. MYC is a nuclear phosphoprotein and functions as a transcription factor. It controls cell cycle progression by simulating G1/S transition and may result in loss of cell cycle arrest and uncontrolled tumor growth when dysfunctional [47]. Other identified significant proteins such as AR, ESR1, SRC, FYN, YWHAQ, YWHAZ, and HSP90AA1 were also shown to contribute to the process of carcinogenesis [48-54].

\section{(2) Cell survival}

The ability of tumor cell populations to expand in number is determined not only by the rate of cell proliferation but also by the rate of cell death [13]. In addition, the acquired resistance of programmed cell death, apoptosis, is a hallmark of cancer. Consequently, proteins annotated with cell survival might be important in carcinogenesis. Twenty-seven significant proteins identified by the network-based biomarker were annotated with cell survival. TP53 (p53) is a well-studied tumor suppressor protein and plays important roles in anti-cancer mechanisms. Its activation is induced by a number of stress signals such as DNA damage, oxidative stress and activated oncogenes. Activated p53 induces cell cycle arrest, apoptosis and inhibition of angiogenesis and metastasis. Once damaged, tumor suppression is severely reduced, resulting in uncontrolled proliferation of the cell. Due to the importance in carcinogenesis, it is no surprise that p53 was found to be significantly mutated in lung adenocarcinoma as well as in squamous cell carcinoma and SCLC $[40,41,55]$. 
TRAF6 functions as a signal transducer in the $\mathrm{NF} \kappa \mathrm{B}$ pathway that activates IKK, in response to proinflammatory cytokines. The identification of TRAF6 by the proposed network-based biomarker approach reinforces the linkage between inflammation and cancer [56,57]. BRCA1 is a nuclear phosphoprotein that contributes to genomic stability. The mutant phenotype of BRCA1 predisposes to breast and ovarian cancer [58]. SP1 is a transcription factor downstream of the TGF $\beta$ pathway and its overexpression contributes to malignant transformation [59]. Other protein kinases, AKT and CSNK2A1 (CK2), were also shown to participate in the carcinogenic process [60-62].

\section{(3) Cell migration}

With the progression of cancer, the malignant tumor cells acquire the ability to migrate and metastasize to distant sites. As a result, the proteins that are relevant to the cell migration capability are crucial for the carcinogenic process. Twenty-three out of 40 significant proteins were annotated with cell migration. PTK2 (FAK), a protein tyrosine kinase in the RTK pathway, is an important mediator within the cell migration process, as well as in cell proliferation and cell survival. Substantial evidence has shown that activated PTK2 leads to tumor growth and metastasis [63], and the level of expression is substantially linked to the invasive potential of tumors [64]. High levels of TSC2 were correlated with increased metastasis and reduced survival in breast cancer patients, revealing a protumorigenic role for TSC2 [65]. The other two significant proteins, PLCG1 and EZR (ezrin), were demonstrated to play critical roles in the metastatic potential of cancer cells but not in primary tumor growth [66,67].

For nine out of the 40 significant proteins identified, little is known about their roles in lung cancer (see Table 1). UBC is identified as a polyubiquitin precursor. Protein ubiquitination is a fundamental, regulatory posttranslational modification controlling intracellular signaling events. It has been associated with protein degradation, DNA repair, cell cycle regulation, endocytosis, and kinase modification [68]. Dysregulation of ubiquitin-mediated signaling is increasingly implicated in some human diseases. Therefore, UBC may be an important target for further characterization of lung carcinogenesis. CALM1 is calmodulin, which mediates the control of a large number of enzymes and other proteins by $\mathrm{Ca}^{2+}$. It is an essential regulator of cell cycle progression and cell survival. Further research is needed to examine its relation with carcinogenesis. PRKACA is a cAMPdependent protein kinase. The identification of PRKACA as a significant protein implies that cAMP signaling might also be involved in lung carcinogenesis. MAPK8IP3 functions as a scaffold protein in the RTK pathway; NCOA6, HGS, and XRCC6 are annotated with cell growth and/or cell survival. However, until now, no empirical evidence has linked their relevance to carcinogenesis, which makes them potential targets for further investigation into lung carcinogenesis.

\section{Diagnostic evaluation of smokers suspect of lung cancer using the network-based biomarker}

The network-based biomarker was constructed based on the primary data set of GSE4115. An independent data set (the prospective data set of GSE4115, 18 smokers with lung cancer and 17 smokers without lung cancer) was then used to evaluate the diagnostic performance of the proposed network-based biomarker. Among the 35 samples, 26 were accurately classified, resulting in an accuracy of $74.29 \%$. The sensitivity and specificity of the proposed approach were also evaluated. The networkbased molecular diagnosis can identify smokers with or without cancer, using a high level of sensitivity of $83.33 \%$, and a moderate specificity of $64.71 \%$ : this enables the proposed network-based biomarker to effectively diagnose the smokers with lung cancer. Further, this approach enables the network-based biomarker to act as a screening test, which, with the aid of other clinical diagnostic tools, both accelerates the process and improves the sensitivity of diagnosis.

The cause of the moderate specificity was further investigated. The difference between the specificity value and the sensitivity value can be attributed to a misclassification of a number of smokers without cancer in the cancer category. The misclassification may be due to similarities in molecular pattern, i.e., the gene expression profiles of smokers with cancer are similar to those of smokers without cancer. In order to validate the hypothesis, Pearson correlation coefficients [30] of gene expression profiles for both smokers with and without cancer were calculated. The mean correlation coefficient of smokers with cancer was 0.9616 , whereas the mean correlation coefficient of smokers without cancer was 0.9441. In addition, the mean correlation coefficient of smokers both with and without cancer (pooled) was as high as 0.9437 , suggesting that the molecular patterns shared among smokers with and without cancer are indeed highly similar. Because of the highly similar molecular patterns and the fact that cigarette smoking is the main instigator of lung cancer, it is likely smokers without cancer could one day develop lung cancer.

In order to validate the predictive performance of the proposed network-based biomarker, several comparisons were made. First, we tested the predictive performance without the information of protein-protein interactions. The 199 differentially expressed proteins selected by ANOVA using the primary data set were used for classification of the prospective data set. A simple hierarchical clustering was performed (see Additional file 3), illustrating that the use of gene expression alone cannot 
accurately classify the prospective data set $(65.71 \%$ accuracy). The comparison shows that the integration of gene expression profiles and protein interaction information can improve lung cancer diagnosis. Second, we compared the predictive performance of the proposed method with randomly selected networks. The average accuracy for 100,000 randomly selected networks of 339 proteins was $48.44 \%$, highlighting the significance of the proposed network-based biomarker. Third, in addition to evaluating the predictive performance using an independent data set, a 5 -fold cross-validation was applied to the primary data set plus the prospective data set. The accuracy of the cross-validation is similar to the initial accuracy of $74.29 \%$ computed using the independent data set, illustrating the robustness and reproducibility of the proposed network-based biomarker approach.

\section{Discussion}

Cancer is a complex disease and carcinogenesis in humans is a multistep process that transforms normal cells into malignant derivatives. Many researchers are investigating the underlying mechanisms that prompt the uncontrolled cell proliferation and metastasis. They have successfully identified some key components of the various steps in the carcinogenesis and some therapeutic interventions have been developed to at least slow down the carcinogenic process. However, because of the complexity, the therapy that targets some specific molecules is only partially effective and tumor-specific. Therefore, investigation of the carcinogenesis from the systems perspective is inevitable. On the other hand, biomarker identification for cancer diagnosis has been a primary research focus in the biomedical field since the use of biomarkers could provide early detection of cancer. As a result, in this study, a network-based biomarker approach has been proposed to simultaneously account for molecular investigation and diagnosis. The proposed approach was applied on the sample data obtained from smokers with and without lung cancer and 40 significant proteins were identified in lung carcinogenesis. The network-based biomarker considers not only differentially expressed proteins but also the protein association network structure. This allows an accurate identification of proteins with low discriminative potentials if such proteins were associated with many other significant proteins [15]. This property is important for the identification of significant proteins in lung carcinogenesis and provides a mechanistic insight into the process. From the mechanism investigation of the 40 significant proteins identified using the network-based biomarker, we found that the significant proteins identified are involved in the pathways that are responsible for cellular processes, including proliferation, differentiation, apoptosis, and metastasis. More importantly, from the results presented, we found that dysregulated signals exist in multiple pathways. There are two possible explanations for the result: the genetic mutations are accumulated respectively to components of different pathways, or the aberrant signals affect different pathways through cross-talk mechanisms. Further investigation is needed to address these two hypotheses. In addition to the investigation of significant proteins, the network-based biomarker can be used as a type of screening test with high sensitivity. Using the same data (the prospective data set of GSE4115), conventional bronchoscopy was shown to be $44 \%$ sensitive to cancer detection [3], which is only half of the proposed network-based biomarker. This further reinforces the clinical utility of the network-based biomarker.

Although our proposed method is shown to be useful, some limitations exist and the need for further improvements remains. In the proposed network-based biomarker approach, gene expression profiles were overlaid to the corresponding proteins for further analysis. However, levels of mRNA do not always correlate with protein levels and do not provide information on posttranslational modification such as phosphorylation that may be critical in regulating protein activity [41]. Consequently, emerging high-throughput proteomic techniques such as protein microarrays would benefit our method by significantly improving the detection performance over mRNA microarray data. In addition, if the genome-wide gene expression levels and protein expression levels can be obtained simultaneously, we are then able to construct the integrated cellular networks of transcription regulations and protein interactions which provide a more integrated network-based biomarker [69]. The protein-protein interaction data from public databases also plays important roles in the proposed method. Nevertheless, there is a large variation in the coverage of protein interaction data across the interaction databases [70]. Therefore, HPRD and BioGRID databases were integrated for the PPI information in this study. We believe that the increased quality and coverage of protein interaction data would enhance the proposed network-based biomarker approach for characterization of lung carcinogenesis. Another limitation of the proposed method was the restriction in the size of the protein association network from the sample size available due to the avoidance of overfitting in the network construction. This results in the exclusion of some well-studied proteins that are relevant to the lung carcinogenic process in the network-based biomarker, including KRAS, MET, PI3KCA. To overcome the problem, more samples are needed. We believe that improvements to diagnostic evaluation using the network-based biomarker lie in the expansion of the 
constructed protein association networks. Many other groups identified the discriminative subnetworks using different methods, especially graph theory-based methods [71,72]. For example, Tian et al. proposed a hypergraph-based iterative learning algorithm for subnetwork identification, which minimizes a cost function under a unified regularization framework [72]. These graphbased methods can also be incorporated to improve the significant protein selection in the proposed method. Further, in this study, the samples for gene expression profiling are simply divided into two groups: smokers with cancer and without cancer. With more sample data, particularly cancer stage-specific samples, we can determine how the network evolves and changes during cancer progression using the proposed method.

Our network-based biomarker provides both a systematic insight into the lung carcinogenic process and a good method for identifying significant proteins, categorized as lung cancer-related proteins and many others that have not been previously reported. These proteins not only provide new targets for further research into understanding the mechanisms of lung carcinogenesis, but are also potential targets for therapeutic interventions. The main challenge of cancer research is to find an effective therapeutic approach that specifically kills malignant cells. Conventional chemotherapy acts by killing all rapidly dividing cells, resulting in toxic effects and damage to normal tissues [73]. With the advances in understanding the mechanisms of the carcinogenic process, the so-called targeted therapy, which is more effective and less harmful to normal cells, is developed to inhibit the specific molecules that play crucial roles in tumor growth. The significant proteins identified by the proposed networkbased biomarker provide suitable molecules to be targeted. For example, gefitinib (Iressa) and erlotinib (Tarceva) are two tyrosine kinase inhibitors that specifically target EGFR. Despite their effectiveness, there are still patients that do not respond well to these drugs [74]. One explanation is that single-target agents are likely to result in network compensation and drug resistance [75]. As a result, multi-target therapeutic interventions that affect multiple targets simultaneously may be required for effective control against cancer. Because biological systems are unable to perform optimally under the influence of two or more simultaneously administered drugs, multi-target therapeutics can prove effective as they may be less vulnerable to adaptive resistance from the human body [76]. With the help of significant proteins identified by the proposed network-based biomarker approach and the pathway information, it is possible the multi-target therapeutic interventions that act on different critical pathways in lung carcinogenesis can be developed.

\section{Conclusions}

Lung cancer is the leading cause of cancer deaths worldwide. Understanding the causes and the underlying mechanisms can help fight the disease. In this study, a network-based biomarker approach, which integrated gene expression profiles and protein interaction information, was developed for molecular investigation and diagnosis for lung cancer. From a systems perspective, the constructed network-based biomarker further evaluated the lung carcinogenic process by use of significant protein identification and diagnostic evaluation. The diagnostic results indicate that the network-based biomarker is sensitive to the diagnosis of smokers with lung cancer and can be used as one kind of screening test. More importantly, the significant proteins identified by the network-based biomarker give mechanistic insights into the carcinogenic process and provide potential therapeutic targets to combat cancer.

\section{Additional material}

\section{Additional file 1: Supplementary Methods \\ Additional file 2: The full names of the significant proteins identified.}

Additional file 3: The hierarchical clustering for 199 differentially expressed proteins of the prospective data set.

\section{Acknowledgements}

We thank Dr. Jhen-Yan Ciou for her valuable discussions. The work was supported by the National Science Council of Taiwan under grants NSC 982627-B-007-014 and NSC 99-2745-E-007-001- ASP.

\section{Authors' contributions}

YCW developed the method, performed the analysis, evaluated the results and wrote the manuscript. BSC provided essential guidance and revised the manuscript. All authors read and approved the final manuscript.

\section{Competing interests}

The authors declare that they have no competing interests.

Received: 29 July 2010 Accepted: 6 January 2011

Published: 6 January 2011

\section{References}

1. Parkin DM, Bray F, Ferlay J, Pisani P: Global cancer statistics, 2002. CA Cancer J Clin 2005, 55:74-108.

2. Kanavos P: The rising burden of cancer in the developing world. Ann Oncol 2006, 17(Suppl 8):viii15-viii23.

3. Spira A, Beane JE, Shah V, Steiling K, Liu G, Schembri F, Gilman S, Dumas YM, Calner P, Sebastiani P, Sridhar S, Beamis J, Lamb C, Anderson T, Gerry N, Keane J, Lenburg ME, Brody JS: Airway epithelial gene expression in the diagnostic evaluation of smokers with suspect lung cancer. Nat Med 2007, 13:361-366.

4. Brambilla E, Travis WD, Colby TV, Corrin B, Shimosato Y: The new World Health Organization classification of lung tumours. Eur Respir J 2001, 18:1059-1068.

5. Khuder SA: Effect of cigarette smoking on major histological types of lung cancer: a meta-analysis. Lung Cancer 2001, 31:139-148.

6. Spira A, Beane J, Shah V, Liu G, Schembri F, Yang X, Palma J, Brody JS: Effects of cigarette smoke on the human airway epithelial cell transcriptome. Proc Natl Acad Sci USA 2004, 101:10143-10148. 
7. Hecht SS: Tobacco smoke carcinogens and lung cancer. J Natl Cancer Inst 1999, 91:1194-1210.

8. Takahashi H, Ogata H, Nishigaki R, Broide DH, Karin M: Tobacco smoke promotes lung tumorigenesis by triggering IKKbeta- and JNK1dependent inflammation. Cancer Cell 2010, 17:89-97.

9. Golub TR, Slonim DK, Tamayo P, Huard C, Gaasenbeek M, Mesirov JP, Coller H, Loh ML, Downing JR, Caligiuri MA, Bloomfield CD, Lander ES: Molecular classification of cancer: class discovery and class prediction by gene expression monitoring. Science 1999, 286:531-537.

10. Alizadeh AA, Eisen MB, Davis RE, Ma C, Lossos IS, Rosenwald A, Boldrick JC, Sabet H, Tran T, Yu X, Powell Jl, Yang L, Marti GE, Moore T, Hudson J Jr, Lu L, Lewis DB, Tibshirani R, Sherlock G, Chan WC, Greiner TC, Weisenburger DD, Armitage JO, Warnke R, Levy R, Wilson W, Grever MR, Byrd JC, Botstein D, Brown PO, et al: Distinct types of diffuse large B-cell lymphoma identified by gene expression profiling. Nature 2000, 403:503-511.

11. Hirsch FR, Merrick DT, Franklin WA: Role of biomarkers for early detection of lung cancer and chemoprevention. Eur Respir J 2002, 19:1151-1158.

12. Ein-Dor L, Zuk O, Domany E: Thousands of samples are needed to generate a robust gene list for predicting outcome in cancer. Proc Natl Acad Sci USA 2006, 103:5923-5928.

13. Hanahan D, Weinberg RA: The hallmarks of cancer. Cell 2000, 100:57-70.

14. Vogelstein B, Kinzler KW: Cancer genes and the pathways they control. Nat Med 2004, 10:789-799.

15. Chuang HY, Lee E, Liu YT, Lee D, Ideker T: Network-based classification of breast cancer metastasis. Mol Syst Biol 2007, 3:140.

16. Chen J, Aronow BJ, Jegga AG: Disease candidate gene identification and prioritization using protein interaction networks. BMC Bioinformatics 2009, 10:73.

17. Nibbe RK, Koyuturk M, Chance MR: An integrative -omics approach to identify functional sub-networks in human colorectal cancer. PLOS Comput Biol 2010, 6:e1000639.

18. Nibbe RK, Markowitz S, Myeroff L, Ewing R, Chance MR: Discovery and scoring of protein interaction subnetworks discriminative of late stage human colon cancer. Mol Cell Proteomics 2009, 8:827-845.

19. Vanunu O, Magger O, Ruppin E, Shlomi T, Sharan R: Associating genes and protein complexes with disease via network propagation. PLOS Comput Biol 2010, 6:e1000641.

20. Taylor IW, Linding R, Warde-Farley D, Liu Y, Pesquita C, Faria D, Bull S, Pawson T, Morris $Q$, Wrana JL: Dynamic modularity in protein interaction networks predicts breast cancer outcome. Nat Biotechnol 2009, 27:199-204.

21. Luscombe NM, Babu MM, Yu H, Snyder M, Teichmann SA, Gerstein M: Genomic analysis of regulatory network dynamics reveals large topological changes. Nature 2004, 431:308-312.

22. Yang SK, Wang YC, Chao CC, Chuang YJ, Lan CY, Chen BS: Dynamic crosstalk analysis among TNF-R, TLR-4 and IL-1R signalings in TNFalphainduced inflammatory responses. BMC Med Genomics 2010, 3:19.

23. Oti M, Snel B, Huynen MA, Brunner HG: Predicting disease genes using protein-protein interactions. J Med Genet 2006, 43:691-698

24. Wang YC, Lan CY, Hsieh WP, Murillo LA, Agabian N, Chen BS: Global screening of potential Candida albicans biofilm-related transcription factors via network comparison. BMC Bioinformatics 2010, 11:53.

25. Stark C, Breitkreutz B-J, Reguly T, Boucher L, Breitkreutz A, Tyers M: BioGRID: a general repository for interaction datasets. Nucl Acids Res 2006, 34 D535-539.

26. Peri S, Navarro JD, Amanchy R, Kristiansen TZ, Jonnalagadda CK, Surendranath V, Niranjan V, Muthusamy B, Gandhi TK, Gronborg M, Ibarrola N, Deshpande N, Shanker K, Shivashankar HN, Rashmi BP, Ramya MA, Zhao Z, Chandrika KN, Padma N, Harsha HC, Yatish AJ, Kavitha MP, Menezes M, Choudhury DR, Suresh S, Ghosh N, Saravana R, Chandran S, Krishna S, Joy M, et al: Development of human protein reference database as an initial platform for approaching systems biology in humans. Genome Res 2003, 13:2363-2371.

27. Johansson R: System modeling and identification. Englewood Cliffs, NJ: Prentice Hall; 1993.

28. Chang YH, Wang YC, Chen BS: Identification of transcription factor cooperativity via stochastic system model. Bioinformatics 2006, 22:2276-2282

29. Akaike $\mathrm{H}$ : A new look at the statistical model identification. IEEE Transactions on Automatic Control 1974, 19:716-723.
30. Pagano M, Gauvreau K: Principles of biostatistics. Pacific Grove, CA: Duxbury; 22000.

31. Vicent S, Lopez-Picazo JM, Toledo G, Lozano MD, Torre W, GarciaCorchon C, Quero C, Soria JC, Martin-Algarra S, Manzano RG, Montuenga LM: ERK1/2 is activated in non-small-cell lung cancer and associated with advanced tumours. Br J Cancer 2004, 90:1047-1052.

32. Hu K, Gan YH, Li SL, Gao Y, Wu DC, Wang CY, Yu GY: Relationship of activated extracellular signal-regulated kinase $1 / 2$ with lung metastasis in salivary adenoid cystic carcinoma. Oncol Rep 2009, 21:137-143.

33. Kyriakis JM, Avruch J: Mammalian mitogen-activated protein kinase signal transduction pathways activated by stress and inflammation. Physiol Rev 2001, 81:807-869.

34. Ventura JJ, Tenbaum S, Perdiguero E, Huth M, Guerra C, Barbacid M, Pasparakis M, Nebreda AR: p38alpha MAP kinase is essential in lung stem and progenitor cell proliferation and differentiation. Nat Genet 2007, 39:750-758.

35. Shi Y, Massague J: Mechanisms of TGF-beta signaling from cell membrane to the nucleus. Cell 2003, 113:685-700.

36. Bierie B, Moses HL: TGF-beta and cancer. Cytokine Growth Factor Rev 2006, 17:29-40.

37. Langenfeld EM, Kong $Y$, Langenfeld J: Bone morphogenetic protein 2 stimulation of tumor growth involves the activation of Smad-1/5. Oncogene 2006, 25:685-692.

38. Zwick E, Bange J, Ullrich A: Receptor tyrosine kinase signalling as a target for cancer intervention strategies. Endocr Relat Cancer 2001, 8:161-173.

39. Langer CJ: Emerging role of epidermal growth factor receptor inhibition in therapy for advanced malignancy: focus on NSCLC. Int J Radiat Oncol Biol Phys 2004, 58:991-1002.

40. Ding L, Getz G, Wheeler DA, Mardis ER, McLellan MD, Cibulskis K, Sougnez C, Greulich H, Muzny DM, Morgan MB, Fulton L, Fulton RS, Zhang Q, Wendl MC, Lawrence MS, Larson DE, Chen K, Dooling DJ, Sabo A, Hawes AC, Shen H, Jhangiani SN, Lewis LR, Hall O, Zhu Y, Mathew T, Ren Y, Yao J, Scherer SE, Clerc K, et al: Somatic mutations affect key pathways in lung adenocarcinoma. Nature 2008, 455:1069-1075.

41. Herbst RS, Heymach JV, Lippman SM: Lung cancer. N Engl J Med 2008, 359:1367-1380.

42. Behrens C, Lin HY, Lee JJ, Raso MG, Hong WK, Wistuba II, Lotan R: Immunohistochemical expression of basic fibroblast growth factor and fibroblast growth factor receptors 1 and 2 in the pathogenesis of lung cancer. Clin Cancer Res 2008, 14:6014-6022.

43. Cox ME, Gleave ME, Zakikhani M, Bell RH, Piura E, Vickers E, Cunningham M, Larsson O, Fazli L, Pollak M: Insulin receptor expression by human prostate cancers. Prostate 2009, 69:33-40.

44. Kishimoto M, Kohno T, Okudela K, Otsuka A, Sasaki H, Tanabe C, Sakiyama T, Hirama C, Kitabayashi I, Minna JD, Takenoshita S, Yokota J: Mutations and deletions of the CBP gene in human lung cancer. Clin Cancer Res 2005, 11:512-519.

45. Barker N, Clevers H: Mining the Wnt pathway for cancer therapeutics. Nat Rev Drug Discov 2006, 5:997-1014.

46. Imai MA, Oda Y, Oda M, Nakanishi I, Kawahara E: Overexpression of E2F1 associated with $\mathrm{LOH}$ at RB locus and hyperphosphorylation of RB in non-small cell lung carcinoma. J Cancer Res Clin Oncol 2004, 130:320-326.

47. Zajac-Kaye M: Myc oncogene: a key component in cell cycle regulation and its implication for lung cancer. Lung Cancer 2001, 34(Suppl 2):S43-46.

48. Chen ZY, Cai L, Bie P, Wang SG, Jiang Y, Dong JH, Li XW: Roles of Fyn in pancreatic cancer metastasis. J Gastroenterol Hepatol 2010, 25:293-301.

49. Vander Griend DJ, D'Antonio J, Gurel B, Antony L, Demarzo AM, Isaacs JT: Cell-autonomous intracellular androgen receptor signaling drives the growth of human prostate cancer initiating cells. Prostate 2010, 70:90-99.

50. Henderson BE, Feigelson HS: Hormonal carcinogenesis. Carcinogenesis 2000, 21:427-433.

51. Byers LA, Sen B, Saigal B, Diao L, Wang J, Nanjundan M, Cascone T, Mills GB, Heymach JV, Johnson FM: Reciprocal regulation of c-Src and STAT3 in non-small cell lung cancer. Clin Cancer Res 2009, 15:6852-6861.

52. Li Z, Zhao J, Du Y, Park HR, Sun SY, Bernal-Mizrachi L, Aitken A, Khuri FR, Fu H: Down-regulation of 14-3-3zeta suppresses anchorage-independent growth of lung cancer cells through anoikis activation. Proc Natl Acad Sci USA 2008, 105:162-167.

53. Wang B, Liu K, Lin HY, Bellam N, Ling S, Lin WC: 14-3-3Tau regulates ubiquitin-independent proteasomal degradation of p21, a novel 
mechanism of p21 downregulation in breast cancer. Mol Cell Biol 2010, 30:1508-1527.

54. Gallegos Ruiz MI, Floor K, Roepman P, Rodriguez JA, Meijer GA, Mooi WJ, Jassem E, Niklinski J, Muley T, van Zandwijk N, Smit EF, Beebe K, Neckers L, Ylstra B, Giaccone G: Integration of gene dosage and gene expression in non-small cell lung cancer, identification of HSP90 as potential target. PLoS One 2008, 3:e0001722.

55. Hollstein M, Sidransky D, Vogelstein B, Harris CC: p53 mutations in human cancers. Science 1991, 253:49-53.

56. Coussens LM, Werb Z: Inflammation and cancer. Nature 2002, 420:860-867.

57. Inoue J, Gohda J, Akiyama T, Semba K: NF-kappaB activation in development and progression of cancer. Cancer Sci 2007, 98:268-274.

58. Welcsh PL, Owens KN, King MC: Insights into the functions of BRCA1 and BRCA2. Trends Genet 2000, 16:69-74

59. Deniaud E, Baguet J, Mathieu AL, Pages G, Marvel J, Leverrier Y: Overexpression of Sp1 transcription factor induces apoptosis. Oncogene 2006, 25:7096-7105.

60. Potter $C$, Pedraza $L G, X u T$ T: Akt regulates growth by directly phosphorylating Tsc2. Nat Cell Biol 2002, 4:658-665.

61. Wang H, Davis A, Yu S, Ahmed K: Response of cancer cells to molecular interruption of the CK2 signal. Mol Cell Biochem 2001, 227:167-174.

62. West KA, Linnoila IR, Belinsky SA, Harris CC, Dennis PA: Tobacco carcinogen-induced cellular transformation increases activation of the phosphatidylinositol 3'-kinase/Akt pathway in vitro and in vivo. Cancer Res 2004, 64:446-451.

63. Mitra SK, Schlaepfer DD: Integrin-regulated FAK-Src signaling in normal and cancer cells. Curr Opin Cell Biol 2006, 18:516-523.

64. McLean GW, Carragher NO, Avizienyte E, Evans J, Brunton VG, Frame MC: The role of focal-adhesion kinase in cancer - a new therapeutic opportunity. Nat Rev Cancer 2005, 5:505-515.

65. Liu H, Radisky DC, Nelson CM, Zhang H, Fata JE, Roth RA, Bissell MJ: Mechanism of Akt1 inhibition of breast cancer cell invasion reveals a protumorigenic role for TSC2. Proc Natl Acad Sci USA 2006, 103:4134-4139.

66. Sala G, Dituri F, Raimondi C, Previdi S, Maffucci T, Mazzoletti M, Rossi C, lezzi M, Lattanzio R, Piantelli M, lacobelli S, Broggini M, Falasca M: Phospholipase Cgamma1 is required for metastasis development and progression. Cancer Res 2008, 68:10187-10196.

67. Deng X, Tannehill-Gregg SH, Nadella MV, He G, Levine A, Cao Y, Rosol TJ: Parathyroid hormone-related protein and ezrin are up-regulated in human lung cancer bone metastases. Clin Exp Metastasis 2007, 24:107-119.

68. Komander D: The emerging complexity of protein ubiquitination. Biochem Soc Trans 2009, 37:937-953.

69. Wang YC, Chen BS: Integrated cellular network of transcription regulations and protein-protein interactions. BMC Syst Biol 2010, 4:20.

70. Mathivanan S, Periaswamy B, Gandhi TK, Kandasamy K, Suresh S, Mohmood R, Ramachandra YL, Pandey A: An evaluation of human protein-protein interaction data in the public domain. BMC Bioinformatics 2006, 7(Suppl 5):S19.

71. Rapaport F, Zinovyev A, Dutreix M, Barillot E, Vert JP: Classification of microarray data using gene networks. BMC Bioinformatics 2007, 8:35.

72. Tian Z, Hwang T, Kuang R: A hypergraph-based learning algorithm for classifying gene expression and arrayCGH data with prior knowledge. Bioinformatics 2009, 25:2831-2838.

73. Petrelli A, Giordano S: From single- to multi-target drugs in cancer therapy: when aspecificity becomes an advantage. Curr Med Chem 2008, 15:422-432.

74. Kris MG, Natale RB, Herbst RS, Lynch TJ Jr, Prager D, Belani CP, Schiller JH, Kelly K, Spiridonidis H, Sandler A, Albain KS, Cella D, Wolf MK, Averbuch SD, Ochs JJ, Kay AC: Efficacy of gefitinib, an inhibitor of the epidermal growth factor receptor tyrosine kinase, in symptomatic patients with non-small cell lung cancer: a randomized trial. JAMA 2003, 290:2149-2158,

75. Erler JT, Linding R: Network-based drugs and biomarkers. J Pathol 2010, 220:290-296

76. Zimmermann GR, Lehar J, Keith CT: Multi-target therapeutics: when the whole is greater than the sum of the parts. Drug Discov Today 2007, 12:34-42.

77. Shannon P, Markiel A, Ozier O, Baliga NS, Wang JT, Ramage D, Amin N, Schwikowski B, Ideker T: Cytoscape: a software environment for integrated models of biomolecular interaction networks. Genome Res 2003, 13:2498-2504.

\section{Pre-publication history}

The pre-publication history for this paper can be accessed here: http://www.biomedcentral.com/1755-8794/4/2/prepub

doi:10.1186/1755-8794-4-2

Cite this article as: Wang and Chen: A network-based biomarker approach for molecular investigation and diagnosis of lung cancer. BMC Medical Genomics 2011 4:2.

\section{Submit your next manuscript to BioMed Central and take full advantage of:}

- Convenient online submission

- Thorough peer review

- No space constraints or color figure charges

- Immediate publication on acceptance

- Inclusion in PubMed, CAS, Scopus and Google Scholar

- Research which is freely available for redistribution

Submit your manuscript at www.biomedcentral.com/submit
Ciomed Central 